\title{
The benefit of hippotherapy for improvement of attention and memory in children with cerebral palsy: A pilot study
}

\author{
Eva Krejčíi,2*, Miroslav Janura ${ }^{1}$, and Zdeněk Svoboda ${ }^{1}$ \\ ${ }^{1}$ Faculty of Physical Culture, Palacký University, Olomouc, Czech Republic; and ${ }^{2}$ The Czech Therapeutic Riding Associa- \\ tion, Prague, Czech Republic
}

Copyright: (C) 2015 E. Krejčí et al. This is an open access article licensed under the Creative Commons Attribution License (http://creativecommons.org/licenses/by/4.0/).

\begin{abstract}
Background: The research is based on an assumption that the hypokinesia induced by cerebral palsy (CP) leads to inhibition of some mental functions. The method we used to treat patients with CP, hippotherapy (HT), is a treatment using horses and belongs to proprioceptive-neuromuscular-facilitative methods. Objective: The aim of the research was to determine the benefit of HT on attention and memory of patients with CP. Methods: There were two groups of patients: short-term HT ( $n=11 ; 8$ females and 3 males, age $15.4 \pm 5.3$ years $)$ and long-term HT $(n=9$; 7 females and 2 males, age $10.8 \pm 2.7$ years). The short-term HT group participated in daily 30 minute-long HT sessions for duration of a week-long summer therapy camp; while with the long-term HT group, HT sessions of the same length were used weekly for 5-6 weeks. Attention and memory skills were assessed with "Numeric square test" and "Verbal learning test", respectively. Both tests were undertaken before starting and after concluding the camp or the 5-6 week long HT period. Results: Attention improvement demonstrated in a significant decrease of an average time of "Numeric square test". The improvement was seen in both long-term HT (15.7 seconds overall average time reduction after HT, $p<.01)$ and short-term HT (20.8 seconds reduction, $p<.01)$ groups. Short-term memory improvement was found in the long-term HT group, demonstrated by a higher number of memorized words (more than half of the patients memorized on average 10.5 more words after HT) $(p<.05)$. While the short-term HT group did not show significant improvement of attention or short-term memory, the average number of memorized words after a diversion of attention and a 30 minute delay increased by 4.1, showing an improvement $(p<.05)$ of long-term memory. Conclusions: Our results suggest that hippotherapy as a part of comprehensive therapy in patients with $\mathrm{CP}$ leads to improvement of memory and attention skill.
\end{abstract}

Keywords: memory, attention, equine-assisted therapy, horse, movement, brain

\section{Introduction}

"Cerebral palsy (CP) describes a group of disorders of the development of movement and posture, causing activity limitation, that are attributed to non-progressive disturbances that occurred in the developing fetal or infant brain" (Bax et al., 2005). The origin was classified as prenatal in $22 \%$, perinatal/neonatal in $47 \%$ and unclassifiable in $31 \%$ (Meberg \& Broch, 2004). CP affects not only motor system, but it often is connected to neurocognitive, sensory and sensitive disorders. One of the main symptoms of this disease

\footnotetext{
* Address for correspondence: Eva Krejčí, Department of Natural Sciences in Kinanthropology, Faculty of Physical Culture, Palacký University, tř. Míru 115, 77111 Olomouc, Czech Republic.E-mail: krejci.ona@gmail.com
}

is spasticity limiting the mobility of individuals within the normal range of movement (Talic \& Honemeyer, 2010). CP requires complex treatment and cooperation of many medical fields (paediatrics, rehabilitation, neurology, and orthopedics, as well as psychology, logopedics, occupational therapy, and special pedagogy) (Komárek, Zumrová, et al., 2008). In order to improve the quality of life of people with $\mathrm{CP}$, it is necessary to apply a very comprehensive and multidisciplinary approach with emphasis on appropriate rehabilitation (Liptak, 2005; Oppenheim, 2009). One of the methods, which may promote modification and reorganization of the central nervous system by affecting multiple systems simultaneously, is hippotherapy (HT) (Jiskrová, Casková, \& Dvořáková, 2010).

The American Hippotherapy Association defines HT as "a physical, occupational, and speech language 
therapy treatment strategy that utilizes equine movement as part of an integrated intervention program to achieve functional outcomes" (Benjamin, 2000). HT belongs to proprioceptive-neuromuscular-facilitative methods; it involves the use of the horse as a therapeutic tool (Shurtleff, Standeven, \& Engsberg, 2009) and could be more successful in improving patients' self-concept than the traditional therapy (Macauley \& Guiterrez, 2004). The rhythmically oscillating back of a horse mainly stimulates a rider's postural reflex mechanisms, resulting in training of balance and coordination (Engel, 2003; Rothaupt, Laser, \& Ziegler, 1998; Strauss, 2000). The main effects of HT are normalization of muscle tone, improved trunk control and walking ability, and increased self-efficacy, confidence, and self-esteem (Debuse, Gibb, \& Chandler, 2009). People with cerebral palsy constitute one of the main user groups of HT (Strauss, 2007).

The research focused on children affected by $\mathrm{CP}$ is based on the assumption, that hypokinesia caused by the disorder leads to an inhibition of some mental functions, particularly attention and memory. The improvement of the musculoskeletal system is an integral part of balance, mobility and independence and can have influence on psychological and cognitive development of patients (Taylor et al., 2009). It is necessary also in other motor functions that are needed for activities of daily living (Zadnikar \& Kastrin, 2011). Regardless of intellectual level, HT could help to remove this inhibition and improve the above listed mental functions.

The aim of the research was to determine the effect of HT on attention and memory of patients with CP.

\section{Methods}

The research was conducted between August 2011 and August 2012, an observations of the patients were made at the summer camps Březejc near Velké Meziřriči and Radíkov near Olomouc, at Piafa HT centre in Vyškov, at Epona HT centre in Brno, and at Ryzáček HT centre in Líšnice. All mentioned HT centres were standardly equipped and equal both in the professional level of the HT teams and suitability of horses used for hippotherapy.

The research design was approved by the Ethics Committee of the Faculty of Physical Culture, Palacký University. All of the legal representatives of the participants were informed about purpose of this study and provided written informed consent prior to data collection.

Two groups of $\mathrm{CP}$ affected patients were examined before starting hippotherapy treatment and after its conclusion. The first group ("short-term HT", $n=11$;
8 females and 3 males, age $15.4 \pm 5.3$ years) conducted daily a 30 minute-long HT session in the one-week rehabilitation camp. Within sensorimotor training unstable platforms were used to imitate the movement of a horse. As a part of therapy Vojta reflex locomotion, Bobath concept, and canis therapy were also used in this camp. In the second group ("long-term HT", $n=9 ; 7$ females and 2 males, age $10.8 \pm 2.7$ years) HT sessions of the same duration were used weekly for a period of 5-6 weeks.

Mental functions were assessed with psycho diagnostic tests. Attention was evaluated with a "Numeric square test" (Jirásek, 1992), when a person under examination pointed numbers on a stimulus chart in the right order ten times. First five times average was labelled M1, next five times average M2 and average of all ten times M. The results were compared to the tables of validated tests with average population of corresponding age and expressed in sten scores - a higher value means better achievement.

Memory functions were assessed with a "Verbal learning test" (Preiss, 1999), when in five attempts fifteen words were read, which the examined person was supposed to repeat. Total number of words memorized for five attempts (I-V) together was used as the test of short-term memory. In other experiments person repeated the words without having them read again, after a diversion of attention (by reading different fifteen words) (VI) and then after thirty minutes (VII). Experiments VI and VII measured quality of long-term memory. In repeated examination at the end of HT treatment, a different set of fifteen words from the retest battery was used.

The data were statistically processed with Statistica (version 10.0; StatSoft, Tulsa, OK, USA). Due to the distribution of the obtained data not being normal (Kolmogorov-Smirnov test), Wilcoxon signed-rank test was used to compare baseline and after treatment measurements of individual groups. For comparison of the obtained data between groups with short-term and long-term HT intervention, Mann-Whitney $U$ test was used. To assess the significance of differences, statistical significance level of $\alpha<.05$ was chosen. The effect size of differences between individual groups was assessed using Cohen's $d$, where the criteria to interpret its magnitude were: $<0.2$ trivial, $0.2-0.5$ small, $0.5-0.8$ moderate, and $>0.8$ large (Cohen, 1988).

\section{Results}

Basic statistical characteristics of measured parameters for memory and attention tests are shown in Table 1. 
Comparison of groups with short-term and long-term HT Before starting the HT treatment, there had been no statistically significant difference found in long-term and short-term HT groups, for any of the measured parameters. After finishing the HT treatment, time average of second five trials of "Numeric square test" was significantly lower for the short-term HT group $(p<.05)$.

\section{The effect of HT intervention}

\section{Long-term HT}

Significant improvement of 10.5 more memorized words $(p<.05 ; d=0.87)$ after HT showed improvement of short-term memory (I-V). The improvement was found in more than a half of patients. Number of memorized words was also higher (1.8 or 2.4 words) after an attention diversion and a 30 minute delay, however differences were not statistically significant.

First five trials time average of "Numeric square test" decreased by 20.1 seconds $(p<.01 ; d=1.24)$. Significant time reduction (10.3 s) was also shown in a second five trials time average $(p<.05 ; d=0.73)$. Both changes resulted in a significant decrease of an overall time average ( $p<.01 ; d=1.41)$. After conversion to the stens, statistically significant improvement was seen in first five trials time average ( $p<.05 ; d=1.42)$ and overall time average $(p<.05 ; d=1.26)$.

\section{Short-term HT}

Comparison of short-term memory tests performed before and after HT intervention showed no statistically significant differences. In long-term memory evaluation (number of words memorized after a 30 minute delay), the number of memorized words increased by 2.7 words $(p<.05 ; d=1.19)$. Similarly, the number of words memorized significantly improved when tested after an attention diversion and a 30 minute delay, this time by 4.1 words ( $p<.05 ; d=0.88$ ).

First five trials time average of "Numeric square test" decreased by 27.1 seconds $(p<.05 ; d=0.56)$. Significant time reduction ( $8.9 \mathrm{~s})$ was also observed in second five trials time average $(p<.01 ; d=0.71)$ and overall time average $(p<.01 ; d=0.80)$. After conversion to the stens, improvement was found in first five trials time average ( 2.6 stens; $p<.05 ; d=1.07$ ), second five trials time average ( 0.9 stens; $p<.05 ; d=0.96$ ), and finally overall time average, which was significantly improved by 1.7 sten $(p<.05 ; d=0.93)$.

\section{Discussion}

We observed the improvement of all measured parameters, with the exception of the number of words memorized in first five trials in the short-term HT group. Level of attention was improved in both measured

Table 1

Measured parameters (Mean $\pm S D$ ) and a significance of the differences in memory and attention evaluation

\begin{tabular}{|c|c|c|c|c|c|c|}
\hline & \multicolumn{3}{|c|}{ Long-term HT } & \multicolumn{3}{|c|}{ Short-term HT } \\
\hline & Before & After & $Z$ & Before & After & $Z$ \\
\hline \multicolumn{7}{|l|}{ Memory } \\
\hline $\mathrm{I}-\mathrm{V}$ & $33.8 \pm 11.6$ & $44.3 \pm 7.8$ & $2.07 *$ & $38.9 \pm 13.3$ & $37.6 \pm 9.9$ & 0.10 \\
\hline VI & $6.3 \pm 2.6$ & $8.1 \pm 2.8$ & 1.86 & $7.9 \pm 2.5$ & $9.3 \pm 3.1$ & 1.54 \\
\hline VII & $6.2 \pm 2.9$ & $8.6 \pm 3.1$ & 1.86 & $7.1 \pm 2.7$ & $9.8 \pm 3.0$ & $2.24 *$ \\
\hline VI + VII & $12.6 \pm 5.5$ & $16.7 \pm 7.2$ & 1.33 & $15.0 \pm 5.0$ & $19.1 \pm 6.1$ & $2.24^{*}$ \\
\hline \multicolumn{7}{|l|}{ Attention } \\
\hline M1 & $91.7 \pm 27.3$ & $71.6 \pm 17.0$ & $2.67 * *$ & $79.4 \pm 71.0$ & $52.3 \pm 33.1$ & $2.13^{*}$ \\
\hline M2 & $61.3 \pm 20.7$ & $51.0 \pm 16.3$ & $1.95^{*}$ & $53.2 \pm 37.9$ & $44.3 \pm 27.6$ & $2.93 * *$ \\
\hline M & $77.0 \pm 21.5$ & $61.3 \pm 16.1$ & $2.67 * *$ & $69.1 \pm 52.9$ & $48.3 \pm 30.1$ & $2.93 * *$ \\
\hline M1S & $2.4 \pm 1.5$ & $3.9 \pm 2.3$ & $2.52 *$ & $2.7 \pm 2.2$ & $5.3 \pm 3.4$ & $2.52 *$ \\
\hline $\mathrm{M} 2 \mathrm{~S}$ & $3.1 \pm 2.1$ & $4.2 \pm 2.9$ & 1.94 & $3.8 \pm 2.8$ & $4.7 \pm 3.3$ & $2.37 *$ \\
\hline MS & $2.8 \pm 1.7$ & $4.0 \pm 2.3$ & $2.52 *$ & $3.3 \pm 2.8$ & $5.0 \pm 3.3$ & $2.49^{*}$ \\
\hline
\end{tabular}

Note. $\mathrm{I}-\mathrm{V}=$ number of words memorized in first the five trials, $\mathrm{VI}=$ number of words memorized after an attention diversion, $\mathrm{VII}=$ number of words memorized after 30 minutes, M1 = first five trials of "Numeric square" test time average (in seconds), M2 = second five trials of "Numeric square" test time average (in seconds), $\mathrm{M}$ = overall "Numeric square" test time average (in seconds), M1S = first five trials of "Numeric square" test time average (in stens), M2S = second five trials of "Numeric square" test time average (in stens), MS $=$ overall "Numeric square" test time average (in stens). ${ }^{*} p<.05, * * p<.01$ 
groups. The only patient, who never exceeded the first sten, was a quadruparetic girl, in which case a severe disability did not allow a comparison to the common population. Pointing to numbers on the stimulus chart was extremely difficult due to limited mobility of the upper limbs. However, if we compared her results in absolute values, she was the patient who achieved the most significant progress and during the second test she was faster by more than a minute. Whereas in the long term HT a short-term memory was improved, in the short term HT this conclusion is valid for the longterm memory.

In HT session nonspecific factors operate, similarly to other physiotherapeutic techniques, for example warmth, tactile stimuli, labyrinthine reflexes etc. (Hollý \& Hornáček, 2005). There are however some other factors which are specific to HT and cannot be replaced. "The goal of HT is not that a patient/client be able to do something better while on the horse but that he or she is able to move better when off the horse" (McGibbon, Andrade, Widener, \& Cintas, 1998). HT may support modification and reorganization of the central nervous system and increase the possibility that these will be used in other movement patterns (Casady \& Nichols-Larsen, 2004). A standardized method of evaluating gross motor functioning in children with $\mathrm{CP}$ is the Gross Motor Function Classification System (McGibbon, Benda, Duncan, \& Silkwood-Sherer, 2009; Palisano et al., 2000; Winchester, Kendall, Peters, Sears, \& Winkley, 2002). A positive effect on the functional motor performance of children with $\mathrm{CP}$ was found in 4 of 5 dimensions after ten weekly HT sessions (Casady \& Nichols-Larsen, 2004). Along with improvements in motor and postural control, HT also can result in increase in quality of life and self-esteem (Bass, Duchowny, \& Llabre, 2009).

Patients participating in our research had to be carefully picked, as for the motor skills evaluation they must have been capable of walking (effect of HT on the motor skills was part of the original research, it is not however a topic of this article); and for the administration of the psycho diagnostic test, patients must have been those of normal intellect, or with just a mild retardation.

The HT can have more than a physical effect - it can have a positive influence on both the social and psychological levels of the patients with neuro-motor disorders. Although there is a substantial amount of research that supports the physical benefits of therapeutic riding, only small evidence exists in relation to its psychological benefits (Ewing, MacDonald, Taylor, \& Bowers, 2007; Kendall, Maujean, Pepping, \& Wright, 2014). A review by Granados and Agís (2011), focusing on children with special needs, concludes that through the combined stimulation of the muscular, skeletal, limbic, vestibular and sensory systems provided by HT, verifiable positive effects can be seen on the psychological and social level and on learning capabilities.

Testament to the effectiveness of HT is the ever growing number of hipporehabilitation centers. HT is used in the treatment of patients suffering from depression and psychotic, anxiety and personality disorders, but has a great effect on children and seniors as well, indirectly influencing the rehabilitation of cognitive functions. In a detailed case study including 24 and later 20 subjects (Taylor et al., 2009) as well as in the study of Kern et al. (2011) the long-term positive effects of HT in autistic subjects were described. Schultz, Remick-Barlow and Robbins (2007) monitored 63 children, who were witnesses or victims of domestic violence. After 19 HT sessions a more distinct effect on those who were victims of domestic violence, abuse or negligence was observed.

Macauley (2006) presented a significant effect of zoo therapy when treating aphasia after brain trauma, where the contact with the animal stimulated the desire and motivation for communication. Frank, McCloskey, and Dole (2011) presented in their case study the results of an 8 week-long HT on a 6 year old girl affected by CP. While no distinct effect on motor functions was noted, there was significant improvement in the patient's self-awareness and social functioning. Many adult users of HT expressed that HT gives them a sense of achievement. The psychological effects of HT enhance its physical effects (Debuse, Gibb, \& Chandler, 2009). Contact with a large animal (horse) may help increase a patient's confidence and self-esteem through its acceptance of him/her as a rider (Meregillano, 2004).

Barca, Frascarelli, and Pezzulo (2012) described visuospatial working memory and visual mental imagery of a child with CP. Moderate impairment of visualmotor integration skills was found as well as cognitive level and memory span. The performance in figures reconstruction, in memorizing matrix patterns and movements along a path was poor.

\section{Limits of study}

The main limit of the study was the small size of the experimental group. The reason for a small size was a low availability of children meeting the above stated criteria for intentional selection. The control group was not created because the number of individuals meeting the required criteria was low. Exclusion of hippotherapy from rehabilitation intervention for part of the experimental group would be unethical. There were several possible interfering variables that could 
have influenced the observed parameters during our research. Possible concurrent rehabilitation and treatment methods might have an effect on improvements of the motor and psychic functions. It is possible in the case of the short-term HT group, where patients were examined at the beginning and after the conclusion of a hippotherapy focused summer camp. Although hippotherapy had a dominant share at this camp, other activities as a canis therapy, various games and camp activities were included in the program of the camp. Other interfering variables could have been psychological and social effects not directly connected to HT treatment, e.g. relations with therapists and other patients, as well as the emotional effect of surroundings etc.

\section{Conclusions}

Results of our study show a positive effect of HT combined with individually defined physiotherapy on improvement of attention and memory functions of patients with CP. The short-term memory was improved in the group with long-term HT. Improvement of attention in this group after ending of HT lesson was characterized by the significant time decrease in "Numeric square test". In the group of short-term HT the number of words in the long-term memory test significantly improved. All measured parameters in the attention test were significantly better after HT sessions.

With regard to the results and in accordance with other referenced studies, it is possible to consider HT as a treatment showing a promise of having complex positive bio-psycho-social effects on the patients with CP.

\section{Acknowledgment}

This study was supported by the internal grant no. FTK_ 2011_027 of the Faculty of Physical Culture, Palacký University, Olomouc "The assessment of the impact of hippotherapy on motor patterns and psychic function re-educating of patients with cerebral palsy". Warm thanks belong to the associations Piafa in Vyškov, Ryzáček o. s. in Líšnice, and EPONA o. s. from Brno for allowing the study to be performed in their stays.

\section{References}

Barca, L., Frascarelli, F., \& Pezzulo, G. (2012). Working memory and mental imagery in cerebral palsy: A single case investigation. Neurocase, 18, 298-304.
Bass, M. M., Duchowny, C. A., \& Llabre, M. M. (2009). The effect of therapeutic horseback riding on social functioning in children with autism. Journal of Autism and Developmental Disorders, 39, 1261-1267.

Bax, M., Goldstein, M., Rosenbaun, P., Leviton, A., Paneth, N., Dan, B., ... Damiano, D. (2005). Proposed definition and classification of cerebral palsy (April 2005). Developmental Medicine and Child Neurology, 47, 571-576.

Benjamin, J. (2000). Introduction to hippotherapy. Retrieved from http://www.americanhippotherapyassociation.org/ aha_hpot_A-intro.htm

Casady, R. L., \& Nichols-Larsen, D. S. (2004). The effect of hippotherapy on ten children with cerebral palsy. Pediatric Physical Therapy, 16, 165-172.

Cohen, J. (1988). Statistical power analysis for the behavioral sciences (2nd ed.). New York, NY: Academic Press.

Debuse, D., Gibb, C., \& Chandler, C. (2009). Effects of hippotherapy on people with cerebral palsy from the users' perspective: A qualitative study. Physiotherapy Theory and Practice, 25, 174-192.

Engel, B. T. (Ed.). (2003). Therapeutic riding II: Strategies for rehabilitation (3rd ed.). Durango, CO: Barbara Engel Therapy Services.

Ewing, C. A., MacDonald, P. M., Taylor. M., \& Bowers, M. J. (2007). Equine-facilitated learning for youths with severe emotional disorders: A quantitative and qualitative study. Child Youth Care Forum, 36, 59-72.

Frank, A., McCloskey, S., \& Dole, R. (2011). Effect of hippotherapy on perceived self-competence and participation in a child with cerebral palsy. Pediatric Physical Therapy, 23, 301-308.

Granados, A., \& Agís, I. (2011). Why children with special needs feel better with hippotherapy sessions: A conceptual review. The Journal of Alternative and Complementary Medicine, 17(3), 35-52.

Hollý, K., \& Hornáček, K. (2005). Hipoterapie: léčba pomocí koně [Hippotherapy: Treatment using the horse]. Ostrava: Montanex.

Jirásek, J. (1992). Č́selný čtverec [Numeric square]. Bratislava: Psychodiagnostika.

Jiskrová, I., Casková, V., \& Dvořáková, T. (2010). Hiporehabilitace [Hipporehabiltiation]. Brno: Mendelova univerzita v Brně.

Kendall, E., Maujean, A., Pepping, C. A., \& Wright, J. J. (2014). Hypotheses about the psychological benefits of horses. Explore: The Journal of Science and Healing, 10, 81-87.

Kern, J. K., Fletcher, C. L., Garver, C. R., Mehta, J. A., Grannemann, B. D., Knox, K. R., ... Trivedi, M. H. (2011). Prospective trial of equine-assisted activities in autism spectrum disorder. Alternative Therapies in Health and Medicine, 17(3), 14-20.

Komárek, V., Zumrová, A., et al. (2008). Dětská neurologie: vybrané kapitoly [Pediatric neurology: Selected chapters] (2nd ed.). Praha: Galén.

Liptak, G. S. (2005). Complementary and alternative therapies for cerebral palsy. Mental Retardation and Developmental Disabilities Research Reviews, 11, 156-163.

Macauley, B. L. (2006). Animal-assisted therapy for persons with aphasia: A pilot study. Journal of Rehabilitation Research and Development, 43, 357-365. 
Macauley, B. L., \& Guiterrez, K. M. (2004). The effectiveness of hippotherapy for children with language-learning disabilities. Communication Disorders Quarterly, 25, 205-217.

McGibbon, N. H., Andrade, C., Widener, G., \& Cintas, H. L. (1998). Effect of an equine-movement therapy program on gait, energy expenditure, and motor function in children with spastic cerebral palsy: A pilot study. Developmental Medicine and Child Neurology, 40, 754-762.

McGibbon, N. H., Benda, W., Duncan, B. R., \& SilkwoodSherer, D. (2009). Immediate and long-term effects of hippotherapy on symmetry of adductor muscle activity and functional ability in children with spastic cerebral palsy. Archives of Physical Medicine and Rehabilitation, 90, 966-974.

Meberg, A., \& Broch, H. (2004). Etiology of cerebral palsy. Journal of Perinatal Medicine, 32, 434-439.

Meregillano, G. (2004). Hippotherapy. Physical Medicine and Rehabilitation Clinics of North America, 15, 843-854.

Oppenheim, W. L. (2009). Complementary and alternative methods in cerebral palsy. Developmental Medicine \& Child Neurology, 51(4), 12-29.

Palisano, R. J., Hanna, S. E., Rosenbaum, P. L., Russell, D. J., Walter, S. D., Wood, E. P., ... Galuppi, B. E. (2000). Validation of a model of gross motor function for children with cerebral palsy. Physical Therapy, 80, 974-985.

Preiss, M. (1999). Pamětový test učení [Verbal learning test]. Bratislava: Psychodiagnostika.

Rothaupt, D., Laser, T., \& Ziegler, H. (1998). Hippoterapia a jej miesto $\mathrm{v}$ rehabilitacii [Hippotherapy and its place in rehabilitation]. Rehabilitácia, 31, 34-37.
Schultz, P. N., Remick-Barlow, G., \& Robbins, L. (2007). Equine-assisted psychotherapy: A mental health promotion/intervention modality for children who have experienced intra-family violence. Health \& Social Care in the Community, 15, 265-271.

Shurtleff, T. L., Standeven, J., \& Engsberg, J. R. (2009). Changes in dynamic trunk/head stability and functional reach after hippotherapy. Archives of Physical Medicine and Rehabilitation, 90, 1185-1195.

Strauss, I. (2007). Hippotherapie - Physiotherapie mit und auf dem Pferd [Hippotherapy: Physiotherapy with and on the horse]. Stuttgart: Thieme.

Talic, A., \& Honemeyer, U. (2010). Cerebral palsy: State of art. Donald School Journal of Ultrasound in Obstetrics and Gynecology, 4, 189-198.

Taylor, R. R., Kielhofner, G., Smith, C., Butler, S., Cahill, S. M., Ciukaj, M. D., \& Gehman, M. (2009). Volitional change in children with autism: A single-case design study of the impact of hippotherapy on motivation. Occupational Therapy in Mental Health, 25, 192-200.

Winchester, P., Kendall, K., Peters, H., Sears, N., \& Winkley, T. (2002). The effect of therapeutic horseback riding on gross motor function and gait speed in children who are developmentally delayed. Physical and Occupational Therapy in Pediatrics, 22(3-4), 37-50.

Zadnikar, M., \& Kastrin, A. (2011). Effects of hippotherapy and therapeutic horseback riding on postural control or balance in children with cerebral palsy: A meta-analysis. Developmental Medicine and Child Neurology, 53, 684-691. 